Commun. Korean Math. Soc. 25 (2010), No. 1, pp. 37-49

DOI 10.4134/CKMS.2010.25.1.037

\title{
GENERALIZED FUZZY NUMBER VALUED BARTLE INTEGRALS
}

\author{
Chun-Kee PARK
}

\begin{abstract}
In this paper we introduce the integration of scalar valued functions with respect to a generalized fuzzy number measure which we call the generalized fuzzy number valued Bartle integral. We first establish some properties of the generalized fuzzy number measures and then study the generalized fuzzy number valued Bartle integrals.
\end{abstract}

\section{Introduction}

The integration of scalar valued functions with respect to a vector measure, which is a special case of the Bartle integral, was first introduced by Bartle [1] in 1956. In general, the Bartle integral is the integration of vector valued functions with respect to a vector measure. As an extension of the special case of the Bartle integral, the integration of scalar valued functions with respect to a set valued measure, which is called the set valued Bartle integral, was first introduced by Papageorgiou [4] in 1985. Wu, Zhang and Wang [6] also studied the set valued Bartle integral. The set valued measure theory was found useful in some of the problems of economics and control theory. In 1996 Xue, Ha and $\mathrm{Wu}[8]$ introduced the fuzzy number measure in Banach spaces, which is called the generalized fuzzy number measure, to generalize the set valued measure theory and studied the relations between generalized fuzzy number measures and set valued measures. As an extension of the set valued Bartle integral we introduce the integration of scalar valued functions with respect to a generalized fuzzy number measure which we call the generalized fuzzy number valued Bartle integral.

In Section 2, we give some concepts and notations which are used in the sequel. In Section 3, we establish some properties of generalized fuzzy number measures. In Section 4, we introduce the generalized fuzzy number valued Bartle integral using the set valued Bartle integral and investigate its properties.

Received October 23, 2007; Revised September 28, 2009.

2000 Mathematics Subject Classification. 26A39, 28B05, 28E10, 46G10.

Key words and phrases. set valued measure, generalized fuzzy number measure, set valued Bartle integral, generalized fuzzy number valued Bartle integral. 


\section{Preliminaries}

Throughout this paper $(X,\|\cdot\|)$ denotes a real separable Banach space with dual $X^{*}$. We write

$P_{0}(X)=\{A: A$ is a nonempty subset of $X\}$,

$P_{b(f)(c)}(X)=\left\{A \in P_{0}(X): A\right.$ is bounded (closed)(convex) $\}$,

$P_{w k c}(X)=\left\{A \in P_{0}(X): A\right.$ is weakly compact and convex $\}$.

For $A \subseteq X$ and $x^{*} \in X^{*}$, let $\sigma\left(x^{*}, A\right)=\sup \left\{x^{*}(x): x \in A\right\}$, the support function of $A$. $\sigma\left(x^{*}, A+B\right)=\sigma\left(x^{*}, A\right)+\sigma\left(x^{*}, B\right)$ and $\sigma\left(x^{*}, \lambda A\right)=\lambda \sigma\left(x^{*}, A\right)$ for each $A, B \in P_{0}(X), x^{*} \in X^{*}$ and $\lambda \geq 0$.

Let $u: X \rightarrow[0,1]$. We denote $[u]^{\alpha}=\{x \in X: u(x) \geq \alpha\}$ for $\alpha \in(0,1] . u$ is called a generalized fuzzy number if for each $\alpha \in(0,1],[u]^{\alpha} \in P_{w k c}(X)$. Let $\mathcal{F}(X)$ denote the set of all generalized fuzzy numbers on $X$.

For $u, v \in \mathcal{F}(X)$ and $\lambda \in \mathbb{R}$, we define $u+v$ and $\lambda u$ as follows:

$$
\begin{gathered}
(u+v)(x)=\sup _{x=y+z} \min (u(y), v(z)), \\
(\lambda u)(x)= \begin{cases}u\left(\frac{1}{\lambda} x\right), & \lambda \neq 0 \\
0, & \lambda=0 .\end{cases}
\end{gathered}
$$

For $u, v \in \mathcal{F}(X)$ and $\lambda \in \mathbb{R},[u+v]^{\alpha}=[u]^{\alpha}+[v]^{\alpha}$ and $[\lambda u]^{\alpha}=\lambda[u]^{\alpha}$ for each $\alpha \in(0,1]$. Hence $u+v, \lambda u \in \mathcal{F}(X)$.

For $A, B \in P_{f}(X)$, let $h(A, B)$ denote the Hausdorff metric of $A$ and $B$ defined by

$$
h(A, B)=\max \left(\sup _{a \in A} d(a, B), \sup _{b \in B} d(b, A)\right),
$$

where $d(a, B)=\inf _{b \in B}\|a-b\|$ and $d(b, A)=\inf _{a \in A}\|a-b\|$. Especially,

$$
h(A, B)=\sup _{\left\|x^{*}\right\| \leq 1}\left|\sigma\left(x^{*}, A\right)-\sigma\left(x^{*}, B\right)\right|
$$

whenever $A, B$ are convex sets. Note that $\left(P_{w k c}(X), h\right)$ is a complete metric space. The number $\|A\|$ is defined by

$$
\|A\|=h(A,\{0\})=\sup _{x \in A}\|x\| .
$$

Define $D: \mathcal{F}(X) \times \mathcal{F}(X) \rightarrow[0,+\infty]$ by the equation

$$
D(u, v)=\sup _{\alpha \in(0,1]} h\left([u]^{\alpha},[v]^{\alpha}\right) \text {. }
$$

Then $D$ is a metric on $\mathcal{F}(X)$.

The norm $\|u\|$ of $u \in \mathcal{F}(X)$ is defined by

$$
\|u\|=D(u, \tilde{0})=\sup _{\alpha \in(0,1]} h\left([u]^{\alpha},\{0\}\right)=\sup _{\alpha \in(0,1]}\left\|[u]^{\alpha}\right\| \text {, where } \tilde{0}=\chi_{\{0\}} .
$$

Let $(\Omega, \Sigma)$ be a measurable space. We say that the $\sigma$-field $\Sigma$ is separable if there exists a sequence $\left(A_{n}\right)_{n=1}^{\infty}$ of elements of $\Sigma$ such that $\Sigma=\sigma\left(\left\{A_{n}: n \geq\right.\right.$ 
1\}). $H: \Sigma \rightarrow P_{0}(X)$ is called a set valued measure if it satisfies the following two conditions:

(1) $H(\phi)=\{0\}$

(2) $H(\cdot)$ is countably additive, i.e., for any sequence $\left(A_{n}\right)_{n=1}^{\infty}$ of pairwise disjoint elements of $\Sigma$ we have that

$$
H\left(\bigcup_{n=1}^{\infty} A_{n}\right)=\sum_{n=1}^{\infty} H\left(A_{n}\right)
$$

where $\sum_{n=1}^{\infty} H\left(A_{n}\right)=\left\{x \in X: x=\sum_{n=1}^{\infty} x_{n}\right.$ (u.c.), $\left.x_{n} \in H\left(A_{n}\right), n \geq 1\right\}$.

Especially, $H: \Sigma \rightarrow P_{w k c}(X)$ is a set valued measure if and only if $\sigma\left(x^{*}, H(\cdot)\right)$ is a scalar valued measure for all $x^{*} \in X^{*}$.

For a set valued measure $H: \Sigma \rightarrow P_{0}(X)$ and $A \in \Sigma$, define $|H|(A)=$ $\sup _{\pi} \sum_{i=1}^{k}\left\|H\left(A_{i}\right)\right\|$, where the supremum is taken over all finite $\Sigma$-partitions $\pi=\left\{A_{1}, A_{2}, \ldots, A_{k}\right\}$ of $A .|H|$ is called the total variation of $H . H$ is said to be of bounded variation if $|H|(\Omega)<\infty$. Note that $|H|$ is a measure.

A vector measure $m: \Sigma \rightarrow X$ is said to be a selector of a set valued measure $H$ if for every $A \in \Sigma, m(A) \in H(A)$. We denote the set of all selectors of $H$ by $S_{H}$. When $H$ is closed valued and of bounded variation, then $S_{H} \neq \phi$. The set of all Bochner integrable functions is denoted by $L^{1}(\Omega, \Sigma, \mu, X)$ or $L^{1}(\mu, X)$, where $(\Omega, \Sigma, \mu)$ is a complete finite measure space.

Definition $2.1([6])$. Let $H: \Sigma \rightarrow P_{0}(X)$ be a set valued measure of bounded variation and let $f: \Omega \rightarrow \mathbb{R}$ be an element of $L^{1}(|H|, \mathbb{R}) . f: \Omega \rightarrow \mathbb{R}$ is said to be Bartle integrable with respect to $H$ if $S_{H} \neq \phi$. In this case, the set valued Bartle integral of $f$ with respect to $H$, denoted by $\int_{\Omega} f(\omega) d H(\omega)$, is defined as follows:

$$
\int_{\Omega} f(\omega) d H(\omega)=\left\{\int_{\Omega} f(\omega) d m(\omega): m \in S_{H}\right\} .
$$

\section{Generalized fuzzy number measures of bounded variation}

In this section, we investigate some properties of generalized fuzzy number measures which are useful for the next section.

Let $(\Omega, \Sigma)$ be a measurable space. $M: \Sigma \rightarrow \mathcal{F}(X)$ is called a generalized fuzzy number measure [8] if it satisfies the following two conditions:

(1) $M(\phi)=\tilde{0}$

(2) $M(\cdot)$ is countably additive, i.e., for any sequence $\left(A_{n}\right)_{n=1}^{\infty}$ of pairwise disjoint elements of $\Sigma$ we have that

$$
M\left(\bigcup_{n=1}^{\infty} A_{n}\right)=\sum_{n=1}^{\infty} M\left(A_{n}\right)
$$

where $\sum_{n=1}^{\infty} M\left(A_{n}\right)$ is defined by

$$
\left(\sum_{n=1}^{\infty} M\left(A_{n}\right)\right)(x)=\sup \left\{\inf _{n} M\left(A_{n}\right)\left(x_{n}\right): x=\sum_{n=1}^{\infty} x_{n} \quad \text { (u.c.) }\right\} \text {. }
$$


Theorem 3.1 ([8]). The mapping $M: \Sigma \rightarrow \mathcal{F}(X)$ is a generalized fuzzy number measure if and only if there exists a family of set valued measure $H^{\alpha}$ : $\Sigma \rightarrow P_{w k c}(X)(\alpha \in(0,1])$ satisfying the following three conditions:

(1) for arbitrary $\alpha, \beta \in(0,1]$ and $A \in \Sigma$, if $\alpha \leq \beta$, then $H^{\alpha}(A) \supseteq H^{\beta}(A)$;

(2) for arbitrary $\alpha_{n} \in(0,1]$ and $A \in \Sigma$, if $\alpha_{n} \uparrow \alpha$, then $H^{\alpha}(A)=$ $\cap_{n=1}^{\infty} H^{\alpha_{n}}(A)$

(3) for arbitrary $A \in \Sigma$, we have

$M(A)(x)= \begin{cases}\sup \left\{\alpha: x \in H^{\alpha}(A), \alpha \in(0,1]\right\}, & \text { if }\left\{\alpha: x \in H^{\alpha}(A), \alpha \in(0,1]\right\} \neq \phi, \\ 0, & \text { if }\left\{\alpha: x \in H^{\alpha}(A), \alpha \in(0,1]\right\}=\phi .\end{cases}$

Note that for a generalized fuzzy number measure $M: \Sigma \rightarrow \mathcal{F}(X)$ the set valued measure $H^{\alpha}: \Sigma \rightarrow P_{w k c}(X)$ is determined by

$$
H^{\alpha}(A)=\{x \in X: M(A)(x) \geq \alpha\} \text {, i.e., } H^{\alpha}(A)=[M(A)]^{\alpha}
$$

for each $\alpha \in(0,1]$ and $A \in \Sigma$.

The following theorem is a version of the representation theorem in [3] where $X$ is $\mathbb{R}^{n}$ instead of a general Banach space.

Theorem $3.2([5])$. If $u \in \mathcal{F}(X)$, then

(1) $[u]^{\alpha} \in P_{w k c}(X)$ for all $\alpha \in(0,1]$,

(2) $[u]^{\alpha} \supseteq[u]^{\beta}$ for $0<\alpha \leq \beta \leq 1$,

(3) if $\left(\alpha_{n}\right) \subseteq[0,1]$ is a nondecreasing sequence converging to $\alpha \in(0,1]$, then $[u]^{\alpha}=\cap_{n=1}^{\infty}[u]^{\alpha_{n}}$.

Conversely, if $\left\{A_{\alpha}: \alpha \in(0,1]\right\} \subseteq P_{0}(X)$ satisfies (1)-(3) above, then there exists $u \in \mathcal{F}(X)$ such that $[u]^{\alpha}=A_{\alpha}$ for each $\alpha \in(0,1]$.

Definition 3.3. Let $M: \Sigma \rightarrow \mathcal{F}(X)$ be a generalized fuzzy number measure. For $A \in \Sigma$, we define $|M|(A)=\sup _{\pi} \sum_{i=1}^{k}\left\|M\left(A_{i}\right)\right\|$, where the supremum is taken over all finite $\Sigma$-partitions $\pi=\left\{A_{1}, A_{2}, \ldots, A_{k}\right\}$ of $A .|M|$ is called the total variation of $M$. We say that $M$ is of bounded variation if $|M|(\Omega)<\infty$.

Theorem 3.4. Let $M: \Sigma \rightarrow \mathcal{F}(X)$ be a generalized fuzzy number measure and let $\left(A_{n}\right)_{n=1}^{\infty}$ be a sequence of elements of $\Sigma$. Then $\left\|\sum_{n=1}^{\infty} M\left(A_{n}\right)\right\| \leq$ $\sum_{n=1}^{\infty}\left\|M\left(A_{n}\right)\right\|$.

Proof. Let $\alpha \in(0,1]$ and let $x \in\left[\sum_{n=1}^{\infty} M\left(A_{n}\right)\right]^{\alpha}$. Then $\left(\sum_{n=1}^{\infty} M\left(A_{n}\right)\right)(x)=$ $\sup \left\{\inf _{n} M\left(A_{n}\right)\left(x_{n}\right): x=\sum_{n=1}^{\infty} x_{n}\right.$ (u.c.) $\} \geq \alpha$.

If $\sup \left\{\inf _{n} M\left(A_{n}\right)\left(x_{n}\right): x=\sum_{n=1}^{\infty} x_{n} \quad\right.$ (u.c.) $\}>\alpha$, then there exists a sequence $\left(x_{n}\right)$ such that $x=\sum_{n=1}^{\infty} x_{n}$ (u.c.) and $\inf _{n} M\left(A_{n}\right)\left(x_{n}\right)>\alpha$. Then $M\left(A_{n}\right)\left(x_{n}\right)>\alpha$ for all $n \in \mathbb{N}$, i.e., $x_{n} \in\left[M\left(A_{n}\right)\right]^{\alpha}$ for all $n \in \mathbb{N}$. Hence $\|x\|=$ $\left\|\sum_{n=1}^{\infty} x_{n}\right\| \leq \sum_{n=1}^{\infty}\left\|x_{n}\right\| \leq \sum_{n=1}^{\infty}\left\|\left[M\left(A_{n}\right)\right]^{\alpha}\right\|$. Thus $\left\|\left[\sum_{n=1}^{\infty} M\left(A_{n}\right)\right]^{\alpha}\right\| \leq$ $\sum_{n=1}^{\infty}\left\|\left[M\left(A_{n}\right)\right]^{\alpha}\right\|$

If $\sup \left\{\inf _{n} M\left(A_{n}\right)\left(x_{n}\right): x=\sum_{n=1}^{\infty} x_{n}\right.$ (u.c.) $\}=\alpha$, then for each $k \in \mathbb{N}$ let $\alpha_{k}=\alpha-\frac{1}{k}$. Choose $k_{0} \in \mathbb{N}$ such that $\alpha_{k_{0}} \in(0,1]$. Then $\alpha_{k} \uparrow \alpha$ as $k \rightarrow \infty$ and for each $k \geq k_{0}$ there exists a sequence $\left(x_{n, k}\right)$ such that $x=$ 
$\sum_{n=1}^{\infty} x_{n, k}$ (u.c.) and $\inf _{n} M\left(A_{n}\right)\left(x_{n, k}\right)>\alpha_{k}$. Then $M\left(A_{n}\right)\left(x_{n, k}\right)>\alpha_{k}$ for all $n \in \mathbb{N}$, i.e., $x_{n, k} \in\left[M\left(A_{n}\right)\right]^{\alpha_{k}}$ for all $n \in \mathbb{N}$. Using the same method as in the above, we have $\left\|\left[\sum_{n=1}^{\infty} M\left(A_{n}\right)\right]^{\alpha}\right\| \leq \sum_{n=1}^{\infty}\left\|\left[M\left(A_{n}\right)\right]^{\alpha_{k}}\right\|$ for each $k \geq k_{0}$. Since $\left[M\left(A_{n}\right)\right]^{\alpha_{k_{1}}} \supseteq\left[M\left(A_{n}\right)\right]^{\alpha_{k_{2}}}$ for $k_{1} \leq k_{2}$ and $\left[M\left(A_{n}\right)\right]^{\alpha}=\cap_{k=k_{0}}^{\infty}\left[M\left(A_{n}\right)\right]^{\alpha_{k}}$ by Theorem 3.2, $\left\|\left[\sum_{n=1}^{\infty} M\left(A_{n}\right)\right]^{\alpha}\right\| \leq \sum_{n=1}^{\infty}\left\|\left[M\left(A_{n}\right)\right]^{\alpha_{k}}\right\|=\sum_{n=1}^{\infty} \| \cap_{i=k_{0}}^{k}$ $\left[M\left(A_{n}\right)\right]^{\alpha_{i}} \|$ for each $k \geq k_{0}$. Letting $k \rightarrow \infty$, we have $\left\|\left[\sum_{n=1}^{\infty} M\left(A_{n}\right)\right]^{\alpha}\right\| \leq$ $\sum_{n=1}^{\infty}\left\|\cap_{i=k_{0}}^{\infty}\left[M\left(A_{n}\right)\right]^{\alpha_{i}}\right\|=\sum_{n=1}^{\infty}\left\|\left[M\left(A_{n}\right)\right]^{\alpha}\right\|$. Hence taking supremum for $0<\alpha \leq 1$, we have $\left\|\sum_{n=1}^{\infty} M\left(A_{n}\right)\right\| \leq \sum_{n=1}^{\infty}\left\|M\left(A_{n}\right)\right\|$.

Theorem 3.5. If $M: \Sigma \rightarrow \mathcal{F}(X)$ is a generalized fuzzy number measure, then $|M|(A)=\sup _{\alpha \in(0,1]}\left|H^{\alpha}\right|(A)$ for all $A \in \Sigma$.

Proof. Let $\alpha \in(0,1], A \in \Sigma$ and let $\pi=\left\{A_{1}, A_{2}, \ldots, A_{k}\right\}$ be a finite $\Sigma$ partition of $A$. If $0<\alpha \leq \beta \leq 1$, then $[M(E)]^{\alpha} \supseteq[M(E)]^{\beta}$ for all $E \in$ $\Sigma$. Hence $\sum_{i=1}^{k} \sup _{\alpha \in(0,1]}\left\|\left[M\left(A_{i}\right)\right]^{\alpha}\right\|=\sup _{\alpha \in(0,1]} \sum_{i=1}^{k}\left\|\left[M\left(A_{i}\right)\right]^{\alpha}\right\|$. Thus we have

$$
\begin{aligned}
|M|(A) & =\sup _{\pi} \sum_{i=1}^{k}\left\|M\left(A_{i}\right)\right\| \\
& =\sup _{\pi} \sum_{i=1}^{k} \sup _{\alpha \in(0,1]}\left\|\left[M\left(A_{i}\right)\right]^{\alpha}\right\| \\
& =\sup _{\pi} \sup _{\alpha \in(0,1]} \sum_{i=1}^{k}\left\|\left[M\left(A_{i}\right)\right]^{\alpha}\right\| \\
& =\sup _{\alpha \in(0,1]} \sup _{\pi} \sum_{i=1}^{k}\left\|\left[M\left(A_{i}\right)\right]^{\alpha}\right\| \\
& =\sup _{\alpha \in(0,1]} \sup _{\pi} \sum_{i=1}^{k}\left\|H^{\alpha}\left(A_{i}\right)\right\| \\
& =\sup _{\alpha \in(0,1]}\left|H^{\alpha}\right|(A) .
\end{aligned}
$$

Note that if a generalized fuzzy number measure $M: \Sigma \rightarrow \mathcal{F}(X)$ is of bounded variation, then the set valued measure $H^{\alpha}: \Sigma \rightarrow P_{w k c}(X)$ is of bounded variation for each $\alpha \in(0,1]$.

Since the supremum of a family of measures is also a measure, we can obtain the following corollary from Theorem 3.5.

Corollary 3.6. If $M: \Sigma \rightarrow \mathcal{F}(X)$ is a generalized fuzzy number measure, then $|M|$ is a measure. 


\section{Generalized fuzzy number valued Bartle integrals}

In this section, we introduce the generalized fuzzy number valued Bartle integral using the set valued Bartle integral and obtain some properties of the integral.

Definition 4.1. Let $M: \Sigma \rightarrow \mathcal{F}(X)$ be a generalized fuzzy number measure of bounded variation and let $f: \Omega \rightarrow \mathbb{R}$ be an element of $L^{1}(|M|, \mathbb{R}) . f: \Omega \rightarrow \mathbb{R}$ is said to be Bartle integrable with respect to $M$ if there exists $\int_{\Omega} f(\omega) d M(\omega) \in$ $\mathcal{F}(X)$ such that

$$
\left[\int_{\Omega} f(\omega) d M(\omega)\right]^{\alpha}=\int_{\Omega} f(\omega) d H^{\alpha}(\omega)
$$

for each $\alpha \in(0,1] . \int_{\Omega} f(\omega) d M(\omega)$ will be called the generalized Bartle integral of $f$ with respect to $M$.

We can obtain the following remark from Theorem 3.2.

Remark 4.2. Let $M: \Sigma \rightarrow \mathcal{F}(X)$ be a generalized fuzzy number measure of bounded variation and let $f: \Omega \rightarrow \mathbb{R}$ be an element of $L^{1}(|M|, \mathbb{R})$. Then $f$ is Bartle integrable with respect to $M$ if and only if $f$ satisfies the following three conditions:

(1) $\int_{\Omega} f(\omega) d H^{\alpha}(\omega) \in P_{w k c}(X)$ for all $\alpha \in(0,1]$,

(2) $\int_{\Omega} f(\omega) d H^{\alpha}(\omega) \supseteq \int_{\Omega} f(\omega) d H^{\beta}(\omega)$ for $0<\alpha \leq \beta \leq 1$,

(3) if $\left(\alpha_{n}\right) \subseteq[0,1]$ is a nondecreasing sequence converging to $\alpha \in(0,1]$, then $\int_{\Omega} f(\omega) d H^{\alpha}(\omega)=\cap_{n=1}^{\infty} \int_{\Omega} f(\omega) d H^{\alpha_{n}}(\omega)$.

Theorem $4.3([6])$. Let $H: \Sigma \rightarrow P_{w k c}(X)$ be a set valued measure of bounded variation and $f \in L^{1}(|H|, \mathbb{R})$. If one of the following two conditions holds:

(1) $\Sigma$ is separable,

(2) $X$ is reflexive,

then $\int_{\Omega} f(\omega) d H(\omega) \in P_{w k c}(X)$.

Theorem 4.4. Let $M: \Sigma \rightarrow \mathcal{F}(X)$ be a generalized fuzzy number measure of bounded variation. If $f: \Omega \rightarrow \mathbb{R}$ is a simple function on $(\Omega, \Sigma)$, then $f: \Omega \rightarrow \mathbb{R}$ is Bartle integrable with respect to $M$.

Proof. Consider the family $\left\{\int_{\Omega} f(\omega) d H^{\alpha}(\omega): \alpha \in(0,1]\right\} \subseteq P_{0}(X)$. By $[6$, Lemma 3.4] $\int_{\Omega} f(\omega) d H^{\alpha}(\omega) \in P_{w k c}(X)$ for all $\alpha \in(0,1]$. Clearly,

$$
\int_{\Omega} f(\omega) d H^{\alpha}(\omega) \supseteq \int_{\Omega} f(\omega) d H^{\beta}(\omega) \text { for } 0<\alpha \leq \beta \leq 1 .
$$

Let $f=\sum_{i=1}^{k} \lambda_{i} \chi_{A_{i}}$ with $A_{1}, A_{2}, \ldots, A_{k} \in \Sigma$ being pairwise disjoint and $\lambda_{1}, \lambda_{2}, \ldots, \lambda_{k} \in \mathbb{R}$. Then just as the proof of Lemma 3.4 in [6] we have

$$
\int_{\Omega} f(\omega) d H^{\alpha}(\omega)=\sum_{i=1}^{k} \lambda_{i} H^{\alpha}\left(A_{i}\right)
$$


for all $\alpha \in(0,1]$. Now let $\left(\alpha_{n}\right)$ be a nondecreasing sequence in $(0,1]$ converging to $\alpha \in(0,1]$. Then we have

$$
\begin{aligned}
\bigcap_{n=1}^{\infty} \int_{\Omega} f(\omega) d H^{\alpha_{n}}(\omega) & =\bigcap_{n=1}^{\infty} \sum_{i=1}^{k} \lambda_{i} H^{\alpha_{n}}\left(A_{i}\right)=\bigcap_{n=1}^{\infty}\left[\sum_{i=1}^{k} \lambda_{i} M\left(A_{i}\right)\right]^{\alpha_{n}} \\
& =\left[\sum_{i=1}^{k} \lambda_{i} M\left(A_{i}\right)\right]^{\alpha}=\sum_{i=1}^{k} \lambda_{i} H^{\alpha}\left(A_{i}\right)=\int_{\Omega} f(\omega) d H^{\alpha}(\omega) .
\end{aligned}
$$

Thus the family $\left\{\int_{\Omega} f(\omega) d H^{\alpha}(\omega): \alpha \in(0,1]\right\}$ satisfies the conditions (1)-(3) of Theorem 3.2. Hence by Theorem 3.2 there exists $u \in \mathcal{F}(X)$ such that $[u]^{\alpha}=\int_{\Omega} f(\omega) d H^{\alpha}(\omega)$ for each $\alpha \in(0,1]$. Therefore $f$ is Bartle integrable with respect to $M$ and $u=\int_{\Omega} f(\omega) d M(\omega)$.

Theorem 4.5. Let $M: \Sigma \rightarrow \mathcal{F}(X)$ be a generalized fuzzy number measure of bounded variation and $f, g \in L^{1}(|M|, \mathbb{R})$. If one of the following two conditions holds:

(1) $\Sigma$ is separable,

(2) $X$ is reflexive,

then

$$
\sup _{\alpha \in(0,1]} h\left(\int_{\Omega} f(\omega) d H^{\alpha}(\omega), \int_{\Omega} g(\omega) d H^{\alpha}(\omega)\right) \leq \int_{\Omega}|f(\omega)-g(\omega)| d|M|(\omega) .
$$

Proof. If $M: \Sigma \rightarrow \mathcal{F}(X)$ is a generalized fuzzy number measure of bounded variation and $f, g \in L^{1}(|M|, \mathbb{R})$, then by Theorem 4.3

$$
\int_{\Omega} f(\omega) d H^{\alpha}(\omega), \int_{\Omega} g(\omega) d H^{\alpha}(\omega) \in P_{w k c}(X) \text { for each } \alpha \in(0,1] .
$$

For each $\alpha \in(0,1]$ and every selector $m$ of $H^{\alpha}$, we have

$$
\begin{aligned}
d\left(\int_{\Omega} f(\omega) d m(\omega), \int_{\Omega} g(\omega) d H^{\alpha}(\omega)\right) & \leq\left\|\int_{\Omega} f(\omega) d m(\omega)-\int_{\Omega} g(\omega) d m(\omega)\right\| \\
& \leq \int_{\Omega}|f(\omega)-g(\omega)| d|m|(\omega) \\
& \leq \int_{\Omega}|f(\omega)-g(\omega)| d\left|H^{\alpha}\right|(\omega) .
\end{aligned}
$$

Since $\left|H^{\alpha}\right|(A) \leq|M|(A)$ for all $A \in \Sigma$ by Theorem 3.5, we have

$$
d\left(\int_{\Omega} f(\omega) d m(\omega), \int_{\Omega} g(\omega) d H^{\alpha}(\omega)\right) \leq \int_{\Omega}|f(\omega)-g(\omega)| d|M|(\omega) .
$$

Changing roles of $f$ and $g$ we also have

$$
d\left(\int_{\Omega} g(\omega) d m(\omega), \int_{\Omega} f(\omega) d H^{\alpha}(\omega)\right) \leq \int_{\Omega}|f(\omega)-g(\omega)| d|M|(\omega) .
$$


Hence we have

$$
\begin{aligned}
& h\left(\int_{\Omega} f(\omega) d H^{\alpha}(\omega), \int_{\Omega} g(\omega) d H^{\alpha}(\omega)\right) \\
&= \max \left(\sup _{m \in S_{H^{\alpha}}} d\left(\int_{\Omega} f(\omega) d m(\omega), \int_{\Omega} g(\omega) d H^{\alpha}(\omega)\right),\right. \\
&\left.\sup _{m \in S_{H^{\alpha}}} d\left(\int_{\Omega} g(\omega) d m(\omega), \int_{\Omega} f(\omega) d H^{\alpha}(\omega)\right)\right) \\
& \leq \int_{\Omega}|f(\omega)-g(\omega)| d|M|(\omega) .
\end{aligned}
$$

Thus we have

$$
\sup _{\alpha \in(0,1]} h\left(\int_{\Omega} f(\omega) d H^{\alpha}(\omega), \int_{\Omega} g(\omega) d H^{\alpha}(\omega)\right) \leq \int_{\Omega}|f(\omega)-g(\omega)| d|M|(\omega) .
$$

Theorem 4.6. Let $M: \Sigma \rightarrow \mathcal{F}(X)$ be a generalized fuzzy number measure of bounded variation and let $f: \Omega \rightarrow \mathbb{R}$ be a bounded $\Sigma$-measurable function. If one of the following two conditions holds:

(1) $\Sigma$ is separable,

(2) $X$ is reflexive,

then $f: \Omega \rightarrow \mathbb{R}$ is Bartle integrable with respect to $M$.

Proof. Consider the family $\left\{\int_{\Omega} f(\omega) d H^{\alpha}(\omega): \alpha \in(0,1]\right\} \subseteq P_{0}(X)$. By Theorem $4.3 \int_{\Omega} f(\omega) d H^{\alpha}(\omega) \in P_{w k c}(X)$ for all $\alpha \in(0,1]$. Clearly, $\int_{\Omega} f(\omega) d H^{\alpha}(\omega) \supseteq$ $\int_{\Omega} f(\omega) d H^{\beta}(\omega)$ for $0<\alpha \leq \beta \leq 1$.

Let $\left(\alpha_{n}\right)$ be a nondecreasing sequence in $(0,1]$ converging to $\alpha \in(0,1]$. Since $f: \Omega \rightarrow \mathbb{R}$ is a bounded $\Sigma$-measurable function, there exists a sequence $\left(f_{k}\right)$ of scalar valued simple functions on $(\Omega, \Sigma)$ such that $\left(f_{k}\right)$ converges to $f$ uniformly on $\Omega$. By Theorem $4.4 f_{k}$ is Bartle integrable with respect to $M$ for each $k \in \mathbb{N}$. Hence $\int_{\Omega} f_{k}(\omega) d M(\omega) \in \mathcal{F}(X)$ for each $k \in \mathbb{N}$. By Theorem 3.2 $\int_{\Omega} f_{k}(\omega) d H^{\alpha}(\omega)=\cap_{n=1}^{\infty} \int_{\Omega} f_{k}(\omega) d H^{\alpha_{n}}(\omega)$ for each $k \in \mathbb{N}$. By Theorem 4.5 we have

$$
\sup _{\alpha \in(0,1]} h\left(\int_{\Omega} f(\omega) d H^{\alpha}(\omega), \int_{\Omega} f_{k}(\omega) d H^{\alpha}(\omega)\right) \leq \int_{\Omega}\left|f(\omega)-f_{k}(\omega)\right| d|M|(\omega)
$$

for each $k \in \mathbb{N}$. 
On the other hand, we have

$$
\begin{aligned}
& h\left(\int_{\Omega} f(\omega) d H^{\alpha}(\omega), \cap_{n=1}^{\infty} \int_{\Omega} f(\omega) d H^{\alpha_{n}}(\omega)\right) \\
\leq & h\left(\int_{\Omega} f(\omega) d H^{\alpha}(\omega), \cap_{n=1}^{\infty} \int_{\Omega} f_{k}(\omega) d H^{\alpha_{n}}(\omega)\right) \\
& \quad+h\left(\cap_{n=1}^{\infty} \int_{\Omega} f_{k}(\omega) d H^{\alpha_{n}}(\omega), \cap_{n=1}^{\infty} \int_{\Omega} f(\omega) d H^{\alpha_{n}}(\omega)\right) \\
= & h\left(\int_{\Omega} f(\omega) d H^{\alpha}(\omega), \int_{\Omega} f_{k}(\omega) d H^{\alpha}(\omega)\right) \\
& \quad+h\left(\cap_{n=1}^{\infty} \int_{\Omega} f_{k}(\omega) d H^{\alpha_{n}}(\omega), \cap_{n=1}^{\infty} \int_{\Omega} f(\omega) d H^{\alpha_{n}}(\omega)\right) \\
\leq & 2 \sup _{\alpha \in(0,1]} h\left(\int_{\Omega} f(\omega) d H^{\alpha}(\omega), \int_{\Omega} f_{k}(\omega) d H^{\alpha}(\omega)\right) \\
\leq & 2 \int_{\Omega}\left|f(\omega)-f_{k}(\omega)\right| d|M|(\omega)
\end{aligned}
$$

for each $k \in \mathbb{N}$. Since $\left(f_{k}\right)$ converges to $f$ uniformly on $\Omega$,

$$
\int_{\Omega}\left|f(\omega)-f_{k}(\omega)\right| d|M|(\omega) \rightarrow 0 \text { as } k \rightarrow \infty
$$

Hence $h\left(\int_{\Omega} f(\omega) d H^{\alpha}(\omega), \cap_{n=1}^{\infty} \int_{\Omega} f(\omega) d H^{\alpha_{n}}(\omega)\right)=0$. So $\int_{\Omega} f(\omega) d H^{\alpha}(\omega)=$ $\cap_{n=1}^{\infty} \int_{\Omega} f(\omega) d H^{\alpha_{n}}(\omega)$. Thus the family $\left\{\int_{\Omega} f(\omega) d H^{\alpha}(\omega): \alpha \in(0,1]\right\}$ satisfies the conditions (1)-(3) of Theorem 3.2. By Theorem 3.2 there exists $u \in \mathcal{F}(X)$ such that $[u]^{\alpha}=\int_{\Omega} f(\omega) d H^{\alpha}(\omega)$ for each $\alpha \in(0,1]$. Hence $f$ is Bartle integrable with respect to $M$ and $u=\int_{\Omega} f(\omega) d M(\omega)$.

Theorem 4.7. Let $M: \Sigma \rightarrow \mathcal{F}(X)$ be a generalized fuzzy number measure of bounded variation, $f, g \in L^{1}\left(|M|, \mathbb{R}_{+}\right)$, where $\mathbb{R}_{+}$is the set of all nonnegative real numbers and let $f$ and $g$ be Bartle integrable with respect to $M$. If one of the following two conditions holds:

(1) $\Sigma$ is separable,

(2) $X$ is reflexive,

then $f+g$ is Bartle integrable with respect to $M$ and

$$
\int_{\Omega}\{f(\omega)+g(\omega)\} d M(\omega)=\int_{\Omega} f(\omega) d M(\omega)+\int_{\Omega} g(\omega) d M(\omega) .
$$

Proof. Since $f$ and $g$ are Bartle integrable with respect to $M, \int_{\Omega} f(\omega) d M(\omega)$, $\int_{\Omega} g(\omega) d M(\omega) \in \mathcal{F}(X)$ and

$$
\left[\int_{\Omega} f(\omega) d M(\omega)\right]^{\alpha}=\int_{\Omega} f(\omega) d H^{\alpha}(\omega), \quad\left[\int_{\Omega} g(\omega) d M(\omega)\right]^{\alpha}=\int_{\Omega} g(\omega) d H^{\alpha}(\omega)
$$


for each $\alpha \in(0,1]$. Since $f, g \in L^{1}\left(|M|, \mathbb{R}_{+}\right)$, by [6, Theorem 3.9] we have

$$
\int_{\Omega}\{f(\omega)+g(\omega)\} d H^{\alpha}(\omega)=\int_{\Omega} f(\omega) d H^{\alpha}(\omega)+\int_{\Omega} g(\omega) d H^{\alpha}(\omega)
$$

for each $\alpha \in(0,1]$. Hence $\int_{\Omega} f(\omega) d M(\omega)+\int_{\Omega} g(\omega) d M(\omega) \in \mathcal{F}(X)$ and

$$
\begin{aligned}
\int_{\Omega}\{f(\omega)+g(\omega)\} d H^{\alpha}(\omega) & =\int_{\Omega} f(\omega) d H^{\alpha}(\omega)+\int_{\Omega} g(\omega) d H^{\alpha}(\omega) \\
& =\left[\int_{\Omega} f(\omega) d M(\omega)\right]^{\alpha}+\left[\int_{\Omega} g(\omega) d M(\omega)\right]^{\alpha} \\
& =\left[\int_{\Omega} f(\omega) d M(\omega)+\int_{\Omega} g(\omega) d M(\omega)\right]^{\alpha}
\end{aligned}
$$

for each $\alpha \in(0,1]$. Hence $f+g$ is Bartle integrable with respect to $M$ and $\int_{\Omega}\{f(\omega)+g(\omega)\} d M(\omega)=\int_{\Omega} f(\omega) d M(\omega)+\int_{\Omega} g(\omega) d M(\omega)$.

We can obtain the following corollary from Theorem 4.6 and Theorem 4.7.

Corollary 4.8. Let $M: \Sigma \rightarrow \mathcal{F}(X)$ be a generalized fuzzy number measure of bounded variation and let $f$ and $g$ be nonnegative bounded $\Sigma$-measurable functions on $\Omega$. If one of the following two conditions holds:

(1) $\Sigma$ is separable,

(2) $X$ is reflexive,

then $f+g$ is Bartle integrable with respect to $M$ and

$$
\int_{\Omega}\{f(\omega)+g(\omega)\} d M(\omega)=\int_{\Omega} f(\omega) d M(\omega)+\int_{\Omega} g(\omega) d M(\omega) .
$$

We can obtain the following corollary from Theorem 4.4 and [6, Lemma 3.4] using a method similar to the proof of Theorem 4.7.

Corollary 4.9. Let $M: \Sigma \rightarrow \mathcal{F}(X)$ be a generalized fuzzy number measure of bounded variation. If $f$ and $g$ are nonnegative simple functions on $(\Omega, \Sigma)$, then $f+g$ is Bartle integrable with respect to $M$ and

$$
\int_{\Omega}\{f(\omega)+g(\omega)\} d M(\omega)=\int_{\Omega} f(\omega) d M(\omega)+\int_{\Omega} g(\omega) d M(\omega) .
$$

Theorem 4.10. Let $M: \Sigma \rightarrow \mathcal{F}(X)$ be a generalized fuzzy number measure of bounded variation, $f \in L^{1}\left(|M|, \mathbb{R}_{+}\right), \lambda \in \mathbb{R}_{+}$and let $f$ be Bartle integrable with respect to $M$. If one of the following two conditions holds:

(1) $\Sigma$ is separable,

(2) $X$ is reflexive,

then $\lambda f$ is Bartle integrable with respect to $M$ and

$$
\int_{\Omega} \lambda f(\omega) d M(\omega)=\lambda \int_{\Omega} f(\omega) d M(\omega) .
$$


Proof. Since $f$ is Bartle integrable with respect to $M$,

$$
\int_{\Omega} f(\omega) d M(\omega) \in \mathcal{F}(X) \text { and }\left[\int_{\Omega} f(\omega) d M(\omega)\right]^{\alpha}=\int_{\Omega} f(\omega) d H^{\alpha}(\omega)
$$

for each $\alpha \in(0,1]$. Since $f \in L^{1}\left(|M|, \mathbb{R}_{+}\right)$and $\lambda f \in L^{1}\left(|M|, \mathbb{R}_{+}\right)$, by $[6$, Theorem 3.9] we have

$$
\begin{aligned}
\sigma\left(x^{*}, \int_{\Omega} f(\omega) d H^{\alpha}(\omega)\right) & =\int_{\Omega} f(\omega) d \sigma\left(x^{*}, H^{\alpha}(\omega)\right), \\
\sigma\left(x^{*}, \int_{\Omega} \lambda f(\omega) d H^{\alpha}(\omega)\right) & =\int_{\Omega} \lambda f(\omega) d \sigma\left(x^{*}, H^{\alpha}(\omega)\right)
\end{aligned}
$$

for each $\alpha \in(0,1]$ and $x^{*} \in X^{*}$. Hence $\lambda \int_{\Omega} f(\omega) d M(\omega) \in \mathcal{F}(X)$ and

$$
\begin{aligned}
\sigma\left(x^{*}, \int_{\Omega} \lambda f(\omega) d H^{\alpha}(\omega)\right) & =\int_{\Omega} \lambda f(\omega) d \sigma\left(x^{*}, H^{\alpha}(\omega)\right) \\
& =\lambda \int_{\Omega} f(\omega) d \sigma\left(x^{*}, H^{\alpha}(\omega)\right) \\
& =\lambda \sigma\left(x^{*}, \int_{\Omega} f(\omega) d H^{\alpha}(\omega)\right) \\
& =\sigma\left(x^{*}, \lambda \int_{\Omega} f(\omega) d H^{\alpha}(\omega)\right)
\end{aligned}
$$

for each $\alpha \in(0,1]$ and $x^{*} \in X^{*}$. Hence

$$
\begin{aligned}
& h\left(\int_{\Omega} \lambda f(\omega) d H^{\alpha}(\omega), \lambda \int_{\Omega} f(\omega) d H^{\alpha}(\omega)\right) \\
= & \sup _{\left\|x^{*}\right\| \leq 1}\left|\sigma\left(x^{*}, \int_{\Omega} \lambda f(\omega) d H^{\alpha}(\omega)\right)-\sigma\left(x^{*}, \lambda \int_{\Omega} f(\omega) d H^{\alpha}(\omega)\right)\right| \\
= & 0
\end{aligned}
$$

for each $\alpha \in(0,1]$. Since $\lambda f \in L^{1}\left(|M|, \mathbb{R}_{+}\right)$, by Theorem $4.3 \int_{\Omega} \lambda f(\omega) d H^{\alpha}(\omega) \in$ $P_{w k c}(X)$ for each $\alpha \in(0,1]$. Since $\left(P_{w k c}(X), h\right)$ is a metric space,

$$
\int_{\Omega} \lambda f(\omega) d H^{\alpha}(\omega)=\lambda \int_{\Omega} f(\omega) d H^{\alpha}(\omega)
$$

for each $\alpha \in(0,1]$. Hence

$$
\begin{aligned}
\int_{\Omega} \lambda f(\omega) d H^{\alpha}(\omega) & =\lambda \int_{\Omega} f(\omega) d H^{\alpha}(\omega) \\
& =\lambda\left[\int_{\Omega} f(\omega) d M(\omega)\right]^{\alpha} \\
& =\left[\lambda \int_{\Omega} f(\omega) d M(\omega)\right]^{\alpha}
\end{aligned}
$$

for each $\alpha \in(0,1]$. Therefore $\lambda f$ is Bartle integrable with respect to $M$ and $\int_{\Omega} \lambda f(\omega) d M(\omega)=\lambda \int_{\Omega} f(\omega) d M(\omega)$. 
We can obtain the following corollary from Theorem 4.6 and Theorem 4.10.

Corollary 4.11. Let $M: \Sigma \rightarrow \mathcal{F}(X)$ be a generalized fuzzy number measure of bounded variation, $\lambda \in \mathbb{R}_{+}$and let $f: \Omega \rightarrow \mathbb{R}$ be a nonnegative bounded $\Sigma$-measurable function. If one of the following two conditions holds:

(1) $\Sigma$ is separable,

(2) $X$ is reflexive,

then $\lambda f$ is Bartle integrable with respect to $M$ and

$$
\int_{\Omega} \lambda f(\omega) d M(\omega)=\lambda \int_{\Omega} f(\omega) d M(\omega) .
$$

Theorem 4.12. Let $M: \Sigma \rightarrow \mathcal{F}(X)$ be a generalized fuzzy number measure of bounded variation and let $f_{n}: \Omega \rightarrow \mathbb{R}(n \in \mathbb{N})$ and $f: \Omega \rightarrow \mathbb{R}$ be Bartle integrable functions with respect to $M$ such that $\left(f_{n}\right)_{n=1}^{\infty}$ is uniformly integrable with respect to $|M|$ and $f_{n}(\omega) \rightarrow f(\omega)|M|$-almost everywhere. If one of the following two conditions holds:

(1) $\Sigma$ is separable,

(2) $X$ is reflexive, then

as $n \rightarrow \infty$.

$$
D\left(\int_{\Omega} f_{n}(\omega) d M(\omega), \int_{\Omega} f(\omega) d M(\omega)\right) \rightarrow 0
$$

Proof. Since $f_{n}(n \in \mathbb{N})$ and $f$ are Bartle integrable with respect to $M$ and so $\int_{\Omega} f_{n}(\omega) d M(\omega), \int_{\Omega} f(\omega) d M(\omega) \in \mathcal{F}(X),(n \in \mathbb{N})$. By Theorem 4.5 and Vitali convergence theorem we have

$$
\begin{aligned}
& D\left(\int_{\Omega} f_{n}(\omega) d M(\omega), \int_{\Omega} f(\omega) d M(\omega)\right) \\
= & \sup _{\alpha \in(0,1]} h\left(\int_{\Omega} f_{n}(\omega) d H^{\alpha}(\omega), \int_{\Omega} f(\omega) d H^{\alpha}(\omega)\right) \\
\leq & \int_{\Omega}\left|f_{n}(\omega)-f(\omega)\right| d|M|(\omega) \rightarrow 0
\end{aligned}
$$

as $n \rightarrow \infty$.

Similarly, we can obtain the following theorem from Theorem 4.5 and the dominated convergence theorem.

Theorem 4.13. Let $M: \Sigma \rightarrow \mathcal{F}(X)$ be a generalized fuzzy number measure of bounded variation and let $f_{n}: \Omega \rightarrow \mathbb{R}(n \in \mathbb{N})$ and $f: \Omega \rightarrow \mathbb{R}$ be Bartle integrable functions with respect to $M$ such that $\left|f_{n}(\omega)\right| \leq f(\omega)$ for all $\omega \in \Omega$ and $f_{n}(\omega) \rightarrow f(\omega)|M|$-almost everywhere. If one of the following two conditions holds:

(1) $\Sigma$ is separable,

(2) $X$ is reflexive, 
then

as $n \rightarrow \infty$.

$$
D\left(\int_{\Omega} f_{n}(\omega) d M(\omega), \int_{\Omega} f(\omega) d M(\omega)\right) \rightarrow 0
$$

\section{References}

[1] R. G. Bartle, A general bilinear vector integral, Studia Math. 15 (1956), 337-352.

[2] J. Diestel and J. J. Uhl Jr., Vector Measures, Math. Surveys, Vol. 15, Amer. Math. Soc., Providence R.I., 1977.

[3] C. V. Negoita and D. A. Ralescu, Application of Fuzzy Sets to Systems Analysis, Wiley, New York, 1975

[4] N. S. Papageorgiou, On the theory of Banach space valued multifunctions. 1. Integration and conditional expectation. 2. Set valued martingales and set valued measures, J. Multivariate Anal. 17 (1985), 185-227.

[5] J. Wu and $\mathrm{C}$. Wu, The w-derivatives of fuzzy mappings in Banach spaces, Fuzzy Sets and Systems 119 (2001), 375-381.

[6] W. Z. Wu, W. X. Zhang, and R. M. Wang, Set valued Bartle integrals, J. Math. Anal. Appl. 255 (2001), 1-20.

[7] X. Xue, M. Ha, and M. Ma, Random fuzzy number integrals in Banach spaces, Fuzzy Sets and Systems 66 (1994), 97-111.

[8] X. Xue, M. Ha, and C. Wu, On the extension of the fuzzy number measures in Banach spaces: Part I. Representation of the fuzzy number measures, Fuzzy Sets and Systems 78 (1996), 347-354.

Department of Mathematics

KANGWON NATIONAL University

Chuncheon 200-701, Korea

E-mail address: ckpark@kangwon.ac.kr 